\title{
Psychologic and sexual dysfunction in primary and secondary infertile male patients
}

\author{
Aytac Sahin ${ }^{1}$, Ahmet Urkmez ${ }^{2}$, Ayhan Verit ${ }^{1}$, Ozgur Haki Yuksel ${ }^{1}$, Fatma Ferda Verit ${ }^{3}$ \\ ${ }^{1}$ Fatih Sultan Mehmet Research \& Training Hospital, Department of Urology, Istanbul, Turkey; \\ ${ }^{2}$ Haydarpasa Numune Research \& Training Hospital, Department of Urology, Istanbul, Turkey; \\ ${ }^{3}$ Suleymaniye Maternity, Research \& Training Hospital, Department of Obstetrics and Gynecology, Center of Assisted \\ Reproduction, Istanbul, Turkey.
}

\begin{abstract}
Summary Objective: The aim of this study was to compare depression and sexual dysfunctions observed in primary and secondary infertile patients. Material and method: The study was performed in 39 primary and 31 secondary infertile male patients. Male sexual health was evaluated using the International Index of Erectile Function (IIEF) score and depression with Beck Depression Inventory (BDI).

Results: Mean age of the participants and their partners were $31.54 \pm 5.37$ (range, 24-48 yrs), and $28.16 \pm 5.58$ (range, 2046 yrs) years, respectively. Mean duration of their marriage ranged between 1 and 17 years (mean, $5.06 \pm 4.15$ yrs). A statistically significant intergroup difference was detected between groups as for BDI scores $(p=0.015 ; p<0.05)$. BDI scores of the primary group were significantly lower than those of the secondary group. A statistically and extremely significant difference was detected between IIEF scores of the groups $(p=0.006 ; p<0.01)$. IIEF scores of the primary infertile group were higher than those of the secondary group. Conclusion: Our study, frequency of the depression and erectile dysfunction seen in the patients with secondary infertility was seen significantly higher than the patients with primary infertility.
\end{abstract}

KEY WORDS: Infertility; Male sexual dysfunction; Depression; Secondary infertile man.

Submitted 2 February 2017; Accepted 23 April 2017

\section{INTRODUCTION}

According to criteria of World Health Organization (WHO) infertility is defined as inability of a sexually active, non-contracepting couple to achieve conception after 12 months of regular sexual intercourse (1). Infertility affects $10-15 \%$ of the couples in their reproductive age. Infertility is related to male and female factors in 40 and $40 \%$ of the cases. Infertility is caused by both male and female factors in $20 \%$ of the cases. The culprit factor cannot be identified (2) in nearly $10-20 \%$ of infertile couples. Failure to become pregnant after 12 months despite regular unprotected sexual intercourse is called primary infertility. Secondary infertility is defined as the inability to become pregnant a time after the birth of one or more biological children. Although there is no clear definition of this time, the 12 months period is also accepted for the secondary infertility by many authors. Diagnosis of infertility can be made in nearly $15 \%$ of the couples who want to have a baby and primary or secondary infertility are detected in 90 and $10 \%$ of them (3). One of the basic and primitive instincts in human beings is reproductive instinct. Reproductive deficiency frequently creates a social stigma, which is perceived as a disgraceful condition (4). Infertility emerges from a sudden and unexpected life crisis, which can be diagnosed in the long run. It results in excessive stressful problems, which strain adjustment mechanisms (5).

Studies analyzing the association between infertility and psychological functions have used one of the following models. One of them is psychological infertility model, which asserts the role of an existing psychopathology on the etiology of infertility and the other model (psychological result) claims that psychological problems arise as a result of infertility (6). Recently direct correlation of mental stress with infertility problems and its impact on the outcomes of infertility treatment has been demonstrated. For example it has been revealed that during natural disasters like earthquakes, sperm quality activities of seminal plasma superoxide dismutase and arginine decrease, and nitric oxide levels increase in men under stress $(7,8)$. Since psychiatric disorders are more frequently analyzed during infertility investigations, scales specific to infertility process haven't been developed yet (9).

In developed countries, it appears to be appropriate to take a detailed history about psychological status of the infertile couple. The rationale of this approach is to predict problems, which might arise during the treatment period and be prepared to confront them. Since patients with severe pathological conditions will affect compliance to treatment, these patients will not be included in this costly and prolonged management process. In this study we analyzed and discussed the possible difference mechanisms of psychologic and sexual dysfunction of primary and secondary infertile male patients.

\section{Material AND METHOdS}

This study was performed in 39 primary and 31 secondary infertile male patients. After institutional review board 
(IRB) approval was received, all patients gave detailed medical history and underwent physical examinations. On physical examination, especially dimensions of both testes, presence of vas deferentia were evaluated. Patients with a history of psychiatric disorders and suspect diagnoses of hepatic, renal, cardiovascular and metabolic diseases, users of drugs affecting prolactin levels including cimetidine and anxiolytic drugs were excluded from the study. At admission, routine seminal analyses of all patients were performed and serum FSH, LH, prolactin and total testosterone levels were checked. All of our patients had olgoasthenozoospermia without varicocele or previous surgery who were under medical treatment and surveillance in our clinic. The patients were questioned as for ejaculatory disorders, decreased libido, erectile dysfunction, depression and anxiety. Male sexual health was evaluated using International Index of Erectile Function (IIEF) Scores and depression with Beck Depression Inventory (BDI). The IIEF form was used for the evaluation, but for the erectile functions, 1, 2, 3, 4, 5 and 15 questions were taken into consideration and the evaluation was made on 30 points.

\section{Statistical analysis}

For statistical analysis NCSS (Number Cruncher Statistical System) 2007\&PASS (Power Analysis and Sample Size) 2008 Statistical Software (Utah, USA) program was used. Study data were evaluated by using descriptive statistical methods and for intergroup comparisons of quantitative data related to parameters with normal or non-normal distribution Student t Test and Mann Whitney U Test were used, respectively. For comparisons of 3 or more than 3 groups with non-normal distribution Kruskal Wallis Test was employed. For the comparison of qualitative data Pearson chi-square test, Fisher's Exact Test, and Yates Continuity Correction Test were utilized. For the evaluation of correlations between parameters, Pearson Correlation Analysis and Spearman's Correlation Analysis were used. Statistical significance was evaluated at a level of $p<0.01$ and $p<0.05$. Finally, multiple logistic regression analysis was used to determine whether sociodemographic characteristics, and depression were associated with male sexual dysfunction. Data were considered significant at $\mathrm{p}<0.05$.

\section{Ethical standard}

This study procedures were reviewed and approved by the Regional Ethical Vetting Board (Istanbul) and have therefore been performed in accordance with the ethical standards laid down in the 1964 Declaration of Helsinki and its later amendments.

\section{RESULTS}

Mean age of the patients and their partners were $31.54 \pm$ 5.37 (range, 24-48 years) and $28.16 \pm 5.58$ (range, 2046 years) years, respectively. Mean duration of their marriage ranged between 1 and 17 years (mean, $5.06 \pm 4.15$ years). BMI measurements of the cases ranged between 20.15 and $37.96 \mathrm{~kg} / \mathrm{m}^{2}$ (mean; $26.09 \pm 3.65 \mathrm{~kg} / \mathrm{m}^{2}$ ). BDI scores of the cases changed between 0 and 26 points (mean; $8.49 \pm 6.79 \mathrm{pts}$ ). IIEF scores of the cases varied between 7 and 30 points (mean; $26.33 \pm 4.61$ pts).
A statistically and extremely significant difference was detected between groups as for age of the cases ( $p=$ $0.001 ; \mathrm{p}<0.01$ ). Ages of the cases in the primary infertile group were significantly lower than the secondary infertile group. Statistically and extremely significant difference was found between groups as for ages of the partners of the infertile male patients $(\mathrm{p}=0.001 ; \mathrm{p}<0.01$ ). Ages of the partners were significantly lower than those of the secondary infertile group. A statistically and extremely significant difference was found between duration of marriage of the cases $(p=0.001 ; p<0.01)$. Cases of the primary infertile group stayed married for significantly shorter periods when compared with the secondary group. BMI measurements of the cases did not demonstrate statistically significant differences between groups ( $p>0.05$ ). A statistically significant difference was detected between groups regarding BDI scores $(\mathrm{p}=$ $0.015 ; \mathrm{p}<0.05$ ). BDI scores of the primary infertile group were significantly lower than those of the secondary group. A statistically, and extremely significant difference was detected between groups regarding IIEF scores $(p=0.006 ; p<0.01)$. IIEF scores of the primary infertile group was significantly higher than the secondary infertile group. The patients' place of residence was either rural $(5.7 \% ; n=4)$ or city $(94.3 \% ; n=66)$ areas. When educational levels of the cases were considered, the cases were either primary school $(31.4 \% ; n=22)$, high school (48.6\%: $\mathrm{n}=54)$ or university $(20 \%$; $\mathrm{n}=14)$. The distribution of monthly income of our cases was low in $18.6 \%(\mathrm{n}=13)$ and moderate to high in $81.4 \%(\mathrm{n}=$ 57). Places of residence and educational level of the cases did not differ significantly between groups ( $p>0.05$ ).

There were positive relationship between male sexual dysfunction (erectile dysfunction) and age $(\mathrm{r}=0.28, \mathrm{p}=$ $0.018)$, partner's age $(r=0.28, p=0.015)$, duration of marriage $(r=0.25, p=0.033)$, depression $(r=0.28, p=$ $0.017)$ and low income $(r=0.28, p=0.016)$. Multivariate logistic regression analysis showed that depression $[\operatorname{Exp}(\beta)=9.8,95 \%$ CI: 1.2- 74.3; $\mathrm{p}=0.027]$ and low income $[\operatorname{Exp}(\beta)=9.8,95 \%$ CI: $1.9-51.3 ; \mathrm{p}=$ $0.007]$ were the independent predictors that were associated with sexual dysfunction. Hosmer-Lemeshow goodness of fit test was found to be well calibrated in this final model $(\mathrm{p}=0.77)($ Table 1$)$.

In primary infertile group, a statistically significant correlation was not detected between BDI scores and ages of the study participants $(p>0.05)$. Between BDI scores and duration of marriages, a statistically significant negative correlation was found at a level of $32.4 \%$ (ie. shorter marriages with increased BDI scores) $(r=-0.324 ; \mathrm{p}=$ 0.044; $\mathrm{p}<0.05$ ). A statistically significant correlation was not found between BDI scores and BMI measurements ( $p>0.05)$. Between BDI and IIEF scores a negative correlation (BDI scores increase with decreasing of IIEF scores) at a level of $32.3 \%$ was detected. $(r=-0.323$; $\mathrm{p}=0.045 ; \mathrm{p}<0 . \mathrm{i05})$. In secondary infertile group, a statistically significant positive correlation (parallel increases in BDI scores and ages) was detected between BDI scores and ages of the participants at a level of $35.8 \%(r=$ $0.358 ; \mathrm{p}=0.048 ; \mathrm{p}<0.05)$. A statistically significant correlation was not found between BDI scores and duration of marriages ( $p>0.05)$. A statistically significant differ- 
Table 1.

Comparative evaluation between groups.

\begin{tabular}{|c|c|c|c|}
\hline Parameters & $\begin{array}{l}\text { Primary infertile men } \\
(\text { mean } \pm S D)(n=39)\end{array}$ & $\begin{array}{l}\text { Secondary infertile men } \\
\text { (mean } \pm \text { SD) }(n=31)\end{array}$ & p \\
\hline Age (y) & $29.2 \pm 3.1$ & $34.3 \pm 6.2$ & $<0.0001$ \\
\hline Partner's age (y) & $25.6 \pm 3.5$ & $31.2 \pm 6.1$ & $<0.0001$ \\
\hline Body mass index $\left(\mathrm{kg} / \mathrm{m}^{2}\right)$ & $25.4 \pm 2.8$ & $26.9 \pm 4.3$ & 0.11 \\
\hline Duration of marriage $(\mathrm{y})$ & $2.5 \pm 1.8$ & $8.1 \pm 4.1$ & $<0.0001$ \\
\hline $\begin{array}{l}\text { Current settlement type } \\
\text { Rural } \\
\text { City }\end{array}$ & $\begin{array}{c}1(2.6 \%) \\
38(97.4 \%)\end{array}$ & $\begin{array}{c}3(9.7 \%) \\
28(90.3 \%)\end{array}$ & 0.20 \\
\hline $\begin{array}{l}\text { Educational status } \\
\text { Primary school } \\
\text { High school } \\
\text { University }\end{array}$ & $\begin{array}{c}13(33.3 \%) \\
20(51.3 \%) \\
6(15.4 \%)\end{array}$ & $\begin{array}{c}9(29.0 \%) \\
14(45.2 \%) \\
8(25.8 \%)\end{array}$ & 0.55 \\
\hline $\begin{array}{l}\text { Monthly income } \\
\text { Low income } \\
\text { Moderate to high income } \\
\text { BECK-D Score } \\
\text { IIEF Score }\end{array}$ & $\begin{array}{c}7(17.9 \%) \\
32(82.1 \%) \\
6.7 \pm 6.0 \\
27.7 \pm 2.1\end{array}$ & $\begin{array}{l}6(19.4 \%) \\
25(80.6 \%) \\
10.6 \pm 7.1 \\
24.4 \pm 6.0\end{array}$ & $\begin{array}{l}0.88 \\
0.017 \\
0.002\end{array}$ \\
\hline
\end{tabular}

Table 2.

Intragroup correlation for age, duration of marriage, BMI and IIEF scores.

\begin{tabular}{|c|c|c|c|c|}
\hline & \multicolumn{4}{|c|}{ BECK-D Scores } \\
\hline & \multicolumn{2}{|c|}{ Primary $(n=39)$} & \multicolumn{2}{|c|}{ Secondary $(n=31)$} \\
\hline & $\mathbf{r}$ & $p$-value & $r$ & $p$-value \\
\hline Age (years) & 0.037 & 0.825 & 0.358 & $0.048^{*}$ \\
\hline Duration of marriage (years) & -0.324 & $0.044 *$ & 0.260 & 0.157 \\
\hline $\mathrm{BMI}\left(\mathrm{kg} / \mathrm{m}^{2}\right)$ & -0.226 & 0.167 & 0.168 & 0.366 \\
\hline IIEF Score & -0.323 & $0.045 *$ & -0.397 & $0.027^{*}$ \\
\hline
\end{tabular}

\section{Table 3.}

Intragroup correlations for IIEF scores, age, duration of marriage, $B M I$ and BECK-D scores.

\begin{tabular}{|c|c|c|c|c|}
\hline & \multicolumn{4}{|c|}{ IIEF Score } \\
\hline & \multicolumn{2}{|c|}{ Primary $(n=39)$} & \multicolumn{2}{|c|}{ Secondary $(n=31)$} \\
\hline & $r$ & p-value & $r$ & $p$-value \\
\hline Age (year) & ${ }^{\mathrm{a}} 0.243$ & 0.135 & a-0.041 & 0.825 \\
\hline Duration of marriage & b0.214 & 0.191 & ${ }^{b}-0.267$ & 0.147 \\
\hline BMI (kg/m2) & a-0.062 & 0.708 & ${ }^{\mathrm{a} 0} 0.054$ & 0.773 \\
\hline BECK-D Score & ${ }^{\mathrm{b}}-0.323$ & $0.045 *$ & ${ }^{b}-0.397$ & $0.027 *$ \\
\hline
\end{tabular}

decreased: $r=-0.323 ; p=0.045 ; p<0.05)$. In secondary infertile group, a statistically significant correlation was not found between IIEF scores and ages of the participants. ( $p>$ 0.05). A statistically significant correlation was not detected between IIEF scores and duration of marriages ( $p>0.05)$. A statistically significant correlation was not detected between IIEF scores and BMI measurements ( $p>0.05$ ). A statistically significant negative correlation was noted between IIEF and BDI scores (IIEF scores increased in parallel with a decrease in BDI scores) at a level of $39.7 \%$ $(\mathrm{r}=-0.397 ; \mathrm{p}=0.027 ; \mathrm{p}<0.05)$ (Table 3$)$. In primary infertile group, BDI and IIEF scores of the cases according to their income level did not significantly differ statistically ( $p$ $>0.05$ ). In secondary infertile group, a statistically significant difference was not found between BDI scores of the cases. ( $p>0.05)$, but IIEF scores of the cases were significantly different statistically in consideration of their income levels $(\mathrm{p}=0.015 ; \mathrm{p}<0.05)$.

\section{Discussion}

Even though erectile dysfunction and ejaculatory disorders manifest themselves as psychogenic effects caused by their inability to have a child, it should not be forgotten that common organic factors may induce infertility itself and psychosexual disorders. In cases where conditions of hormonal imbalance, like prolactinoma or primary hypogonadism as cause infertility, this endocrinologic disorder is also the etiological factor of associated sexual dysfunction. Therefore, before resorting to psychogenic factors, organic causes should be ruled out. In the differential diagnosis process in infertile couples, complete anamnesis, physical examination, hormonal evaluation and if required nocturnal penile tumescence test should be used. In our study the patients observed in our clinic with the diagnosis of infertility were interrogated with respect to sexual and ejaculatory dysfunction. The patients with organic disorders and, in consid-

ence was not detected between BDI scores and BMI measurements $(p>0.05)$. A statistically significant negative correlation was noted between BDI and IIEF scores (BDI scores increased concurrently with a decrease in IIEF scores $)$ at a level of $39.7 \%(r=-0.397$; $p=0.027$; p < 0.05) (Table 2).

In primary infertile group a statistically significant correlation was not found between IIEF scores and ages of the participants scores $(p>0.05)$. A statistically significant correlation was not found between IIEF scores and duration of marriages ( $p>0.05$ ). A statistically significant correlation was not found between IIEF scores and BMI measurements ( $p>0.05$ ). A statistically significant negative correlation was noted between IIEF and BDI scores at a level of $32.3 \%$ (IIEF scores increased as BDI scores eration of surgical stress, those who had undergone any surgical intervention were excluded from the study.

Older theories related to infertility focused on psychological factors as cause of infertility, however during 1970s, Manning's psychological strain theory revealed that psychological problems were not the cause but the effect of infertility. Infertility is a stressful life experience and depressive symptoms are normal manifestations of reactive behaviors (10). In nearly $37 \%$ of infertile women manifest depressive symptoms 2 times more frequently relative to the control group as evaluated with BDI (11). In another similar study, the Authors detected mildmoderate $(28 \%)$, moderate-severe $(7 \%)$ or very severe (1\%) degrees of depression in infertile women (12). In a separate study 2 of 3 of the infertile women had com- 
plaints of anxiety (13). In a similar study by Oddens et al., the Authors detected depressive disease in a quarter of their patients (14). In an anxiety survey study performed on 130 infertile women in China, the results obtained demonstrated that $83 \%$ of infertile women had emotional disorder of various degrees and $25 \%$ of them demonstrated moderate or severe type of anxiety. Global incidence rates of depressive disorders and anxiety among infertile women range between 24 and 36\% and 67$84 \%$, respectively (15). In some studies, Authors have demonstrated that infertile men had demonstrated better emotional function index scores relative to women. It is not known whether this observation might be due to introverted nature of men on this issue. One of the most important criteria seen among women is that emotional statuses of infertile women are more deeply affected than infertile men (16).

A study analyzed the impact of psychological stress on male hormones and sperm quality of male partners. Seventy infertile male partners were investigated as for psychological stress levels using Anxiety and Depression Scale scoring system and with measurements of total serum testosterone, serum GnRH, FSH, LH levels and spermiograms. The researchers indicated that psychological stress firstly lowers serum total testosterone levels, and secondarily increases serum LH and FSH levels, which suggest that stress management might have an important place in male infertility (17). We included only infertile male patients in our study and found depression rates in primary and secondary infertile groups as 22 and 38\%, respectively. ED rates in primary and secondary infertile groups were 15 and $45 \%$ respectively. All of our patients had normal levels of FSH, LH, and testosterone. Similar study that was conducted on infertile women with primary and secondary infertility and in parallel with an our previous study, sexual dysfunction in infertile patients with secondary infertility was seen significantly higher than the patients with primary one (18). In a study that investigated association between ED and socio-economic parameters, lowincome was found a predictive parameter (19). In our study, multivariate logistic regression analysis showed that depression and low income were independent predictors that were associated with sexual dysfunction. Long-lasting infertility, and failed treatment attempts aggravate psychological problems as stress and especially depression (20). In infertile women depression and anxiety worsen with advanced age and prolonged duration of infertility (21). In our study, duration of infertility differed statistically with extreme significance between groups. Duration of infertility is significantly shorter in the primary infertile group when compared with the secondary infertile group. A statistically significant difference was detected between groups regarding BDI scores of the cases. BDI scores of the primary infertile group was significantly lower than those of the secondary infertile group.

On the other hand immunological investigations on psychoneurosis have demonstrated the potential impact of psychological factors on immunity $(22,23)$.

For instance, natural killer cell (NK) activity has been determined as the main factor in premature termination of pregnancy $(24,25)$. Provided that these studies have taken psycho-neuro-endocrine-immune system into consideration, the authors indicated that stress induced by infertility concerns would worsen the psychological status of the couple, and objective evaluation of emotional reactions of the couples to infertility, and appropriate psychosomatic approach might be as fruitful as pharmacological therapy of infertility.

Limbic system is considered as 'emotional brain'. Every type of mental exercise has demonstrated an increase in hippocampal volume and neurogenesis. Besides, sustained stressful conditions cause a decrease in hippocampal volume and neurogenesis of hippocampal neurons (26). As an adaptive response to stress, blood cortisol levels peak suddenly. However excessive and prolonged cortisol release creates a neurotoxic effect on brain leading to decreases in synaptic plasticity and neurogenesis and hippocampal atrophy. Probably these deleterious effects deteriorate memory and cognitive functions with resultant sexual dysfunction. In depressive states, a correlation has been indicated between hippocampal atrophy, plasma cortisol levels, and duration of depression. Resolution of atrophy has been reported in line with amelioration of depression (27).

In some studies performed, the Authors stated that a negative correlation between depression and testosterone in men and cortisol in women may be associated with biological pathophysiology of male and female depression, respectively (28).

Adrenal androgens, dehydroepiandrosterone (DHEA) and its sulfate derivative are known to play important roles in controlling emotions, mood and anxiety. A relevant study has demonstrated that in male patients, serum DHEA-S levels decreased significantly when compared with the male control subjects, however in infertile female patients an important difference was not observed relative to the control group.

Since DHEA therapy was effective treatment for depression, increased levels of serum DHEA can be associated with biological pathophysiologic mechanisms of depression. However, DHEA/DHEA-S levels have been reported as a weak marker of the severity of depression (29).

In our study, we observed that secondary infertile group was more negatively affected in terms of BDI and IIEF scores. When the groups were evaluated separately; duration of marriage was negatively correlated with BDI in primary infertile group, on the other hand patient age and low-income were positively correlated with BDI in secondary group. We think that the secondary infertility patients felt extra pressure on their moods because they had already achieved the fertility award at least once in their previous life but they could not manage this again, however all intra or extra motivations directed at this focus. Our study was the pioneer one, which was conducted on the infertile men with primary and secondary infertility in regard to find out the differences of psychologic and sexual dysfunction patterns of both groups. For a successful infertility management, elimination of psychological stress factors might be required. In certain patient groups, treatment of sexual dysfunction may achieve fertility and eliminate infertility related problems. However, we are of the opinion that as a study 
design, men and women factors should be evaluated together in infertile pairs and we think that our subject study will make a greatest contribution to the literature.

\section{REFERENCES}

1. World Health Organization. WHO Manual for the standardized investigation and diagnosis of the infertile couple. Cambridge: Cambridge University Press, 1999.

2. Freidman T. Infertility and assisted reproduction. Bailiere's Clinical Obst. andGyne. Scandinavica. 1989; 66:517-21.

3. EAU Guidelines on Male Infertility, 2010.

4. Herz EK. Infertility and bioethical issues the new reproductive technologies. The Psychiatric Clinics of North America. 1989; 12:117-31.

5. Forrest L, Gilbert LG. Infertility: an unanticipated and prolonger life crisis. J Mental Health Counsel. 1992; 14:42-58.

6. Berg BJ, Wilson JF. Psychiatric morbidity in infertile population. Fertility and Sterility. 1990; 53:654-61.

7. Collodel G, Moretti E, Fontani V, et al. Effect of emotional stress on sperm quality. Indian J Med Res. 2008; 128:254-61.

8. Abu-Musa AA, Nassar AH, Hannoun AB, et al. Effect of the Lebanese civil war on sperm parameters. FertilSteril. 2007; 88:1579-1582

9. Greil AL, McQuillan J, Lowry M, Shreffler KM. Infertility treatment and fertility-specific distress: a longitudinal analysis of a population-based sample of U.S. Women Soc Sci Med. 2011; 73: 87-94.

10. Wroolie TE, Williams KE, Keller J, et al. Mood and neuropsychological changes in women with midlife depression treated with escitalopram. J Clin. Psychopharmacol. 2006; 26:361-6.

11. Domar AD, Broome A, Zuttermeister PC, et al. The prevalence and predictability of depression in infertile women. Fertil Steril. 1992; 58:1158-63

12. Thiering $P$, Beaurepaire J, Jones $M$, et al. Mood state as a predictor of treatment outcome after in vitro fertilization/embryo transfer technology. J Psychosom Res. 1993; 37:481-91.

13. Guerra D, Liobra A, Veiga A, et al. Psychiatric morbidity in couples attending a fertility service. Hum. Reprod. 1998; 13:1733-6.

14. Oddens BJ, Tonkelaar ID, Nieuwenhuyse H. Psychosocial experience in women facing fertility problems a comparative survey. Hum.Reprod. 1999; 14:255-61.

15. Lu Y, Yang L, Lu G. Mental status and personality of infertile women. Zhonghua Fu Chan KeZaZhi 1995; 30:34-7.
16. Franco JG Jr, RazeraBaruffi RL, Mauri AL, et al. Psychological evaluation test for infertile couples. J Assist Report Genet. 2003; 19:269-73.

17. Bhongade MB, Prasad S, Jiloha RC, et al. Effect of psychological stress on fertility hormones and seminal quality in male partners of infertile couples. Andrologia. 2015; 47:336-42.

18. Keskin U, Coksuer H, Gungor S, et al. Differences in prevalence of sexual dysfunction between primary and secondary infertile women. Fertil Steril. 2011; 96:1213-7.

19. Laumann EO, Paik A, Rosen RC. Sexualdysfunction in the United States: prevalenceandpredictors.JAMA. 1999; 281:537-44.

20. Lok IH, Lee DT, Gheung LP, et al. Psychiatric morbidity amongst infertile Chinese women undergoing treatment with assisted reproductive technology and the impact of treatment failure. Gynecol. Obstet. Invest. 2002; 53:195-9.

21. Guz H, Ozkan A, Sarisoy G, et al. Psychiatric symptoms in Turkish infertil women. J Psychosom Obstet Gynaecol. 2003; 24:267-71

22. Strauman TJ, Lemieux AM, Coe CL. Self-discrepancy and natural killer cell activity: Immunological consequences of negative selfevaluation. J Pers Soc Psychol. 1993; 64:1042-52.

23. Ishihara S, Nohara R, Makita S, et al. Immune function and psychological factors in patients with coronary heart disease. Jpn Circ J. 1999; 63:704-9.

24. Aoki K, Kajiura S, Matsumoto Y, et al. Preconceptional naturalkiller-cell activity as a predictor of miscarriage. Lancet. 1995; 345:1340-2.

25. Matsubayashi H, Hosaka T, Sugiyama Y, et al. Increased natural killer-cell activity is associated with infertile women. Am J Reprod Immunol. 2001; 46:318-22.

26. Czeh B, Michaelis T, Watanabe T, et al. Stress-induced changes in cerebral metabolites, hippocampal volume, and cell proliferation are prevented by antidepressant treatment with tianeptine. Proc Natl Acad Sci. USA. 2001; 98:12796-801.

27. Spolsky RM. Glucocorticoids and hippocampal atrophy in neuropsychiatric disorders. Arc Gen Psychiatry. 2000; 57:925-35.

28. Wischmann T. Sexual disorders in infertile couples: an update. Curr Opin Obstet Gynecol. 2013; 25:220-2.

29. Matsuzaka H, Maeshima H, Kida S, et al. Gender differences in serum testosterone and cortisol in patients with major depressive disorder compared with controls. Int J Psychiatry Med. 2013; 46:203-11.

\section{Correspondence}

Aytac Sahin, MD

Ayhan Verit, MD

Ozgur Haki Yuksel, MD

Fatih Sultan Mehmet Research \& Training Hospital, Department of Urology, Istanbul, Turkey

Ahmet Urkmez, MD (Corresponding Author)

ahmeturkmez@hotmail.com

HaydarpasaNumune Research and Training Hospital, Dept. of Urology,

Uskudar, Tr- 34668 Istanbul, Turkey

Fatma Ferda Verit, MD

ffverit@gmail.com

Suleymaniye Maternity, Research \& Training Hospital, Department of

Obstetrics and Gynecology, Center of Assisted Reproduction, Istanbul, Turkey 